\title{
The inverse Fueter mapping theorem in integral form using spherical monogenics
}

\author{
Fabrizio Colombo \\ Politecnico di Milano \\ Dipartimento di Matematica \\ Via Bonardi, 9 \\ 20133 Milano, Italy \\ fabrizio.colombo@polimi.it
}

\author{
Irene Sabadini \\ Politecnico di Milano \\ Dipartimento di Matematica \\ Via Bonardi, 9 \\ 20133 Milano, Italy \\ irene.sabadini@polimi.it
}

\author{
Franciscus Sommen \\ Clifford Research Group \\ Faculty of Sciences \\ Ghent University \\ Galglaan 2, 9000 Gent, Belgium \\ Frank.Sommen@UGent.be
}

\begin{abstract}
In this paper we prove an integral representation formula for the inverse Fueter mapping theorem for monogenic functions defined on axially symmetric open sets $U \subseteq \mathbb{R}^{n+1}$, i.e. on open sets $U$ invariant under the action of $S O(n)$. Every monogenic function on such an open set $U$ can be written as a series of axially monogenic functions of degree $k$, i.e. functions of type $\breve{f}_{k}(x):=\left[A\left(x_{0}, \rho\right)+\underline{\omega} B\left(x_{0}, \rho\right)\right] \mathcal{P}_{k}(\underline{\omega})$, where $A\left(x_{0}, \rho\right)$ and $B\left(x_{0}, \rho\right)$ satisfy a suitable Vekua-type system and $\mathcal{P}_{k}(\underline{\omega})$ are spherical monogenic polynomials of degree $k$. The Fueter mapping theorem says that given a holomorphic function $f$ of a paravector variable defined on $U$ then the function $\breve{f}(x) \mathcal{P}_{k}(\underline{x})$ given by

$$
\Delta^{k+\frac{n-1}{2}}\left(f(x) \mathcal{P}_{k}(\underline{x})\right)=\breve{f}(x) \mathcal{P}_{k}(\underline{x})
$$

is a monogenic function. The aim of this paper is to invert the Fueter mapping theorem by determining a holomorphic function $f$ of a paravector variable in terms of $\breve{f}(x) \mathcal{P}_{k}(\underline{x})$. This result allows to invert the Fueter mapping theorem for any monogenic function defined on an axially symmetric open set.
\end{abstract}

Key words: The inverse Fueter mapping theorem in integral form, Fueter primitives, elliptic equations, Cauchy-Riemann equations, Vekua-type system.

Mathematical Review Classification numbers: 30G35, 32A25, 30E20.

\section{Introduction and notations}

The Fueter mapping theorem, see [14], is an ingenious tool to generate Cauchy-Fueter regular functions from holomorphic functions of a variable in the upper complex plane. Such a theorem has been extended in order to obtain monogenic functions (see [1], [4], [15], [18]), by Sce [25] for $n$ odd and to Qian [22] in the general case. Later on, Fueter's theorem has been generalized to the case in which a function $f$ as above is multiplied by a monogenic homogeneous polynomial 
of degree $k$, (see [19], [20], [21], [26]) and to the case in which the function $f$ is defined on an open set $U$ not necessarily chosen in the upper complex plane, (see [22], [23], [24]).

The setting in which we work is the real Clifford algebra $\mathbb{R}_{n}$ over $n$ imaginary units $e_{1}, \ldots, e_{n}$ satisfying the relations $e_{i} e_{j}+e_{j} e_{i}=-2 \delta_{i j}$. An element in the Clifford algebra will be denoted by $\sum_{A} e_{A} x_{A}$ where $A=i_{1} \ldots i_{r}, i_{\ell} \in\{1,2, \ldots, n\}, i_{1}<\ldots<i_{r}$, is a multi-index, $e_{A}=e_{i_{1}} e_{i_{2}} \ldots e_{i_{r}}$ and $e_{\emptyset}=1$. As it is well known, $\mathbb{R}_{1}$ is the algebra of complex numbers $\mathbb{C}$ (the only case in which the Clifford algebra is commutative), while for $n=2$ we obtain the division algebra of real quaternions $\mathbb{H}$. For $n>2$, the Clifford algebras $\mathbb{R}_{n}$ have zero divisors. In $\mathbb{R}_{n}$, we can identify some specific elements with the vectors in the Euclidean space $\mathbb{R}^{n}$ : an element $\left(x_{1}, x_{2}, \ldots, x_{n}\right) \in \mathbb{R}^{n}$ can be identified with a so called 1-vector in the Clifford algebra through the map $\left(x_{1}, x_{2}, \ldots, x_{n}\right) \mapsto \underline{x}=x_{1} e_{1}+\ldots+x_{n} e_{n}$.

An element $\left(x_{0}, x_{1}, \ldots, x_{n}\right) \in \mathbb{R}^{n+1}$ will be identified with the element $x=x_{0}+\underline{x}$ called, in short, paravector. The norm of $x \in \mathbb{R}^{n+1}$ is defined as $|x|^{2}=x_{0}^{2}+x_{1}^{2}+\ldots+x_{n}^{2}$. The real part $x_{0}$ of $x$ will be also denoted by $\operatorname{Re}[x]$. A function $f: U \subseteq \mathbb{R}^{n+1} \rightarrow \mathbb{R}_{n}$ is seen as a function $f(x)$ of the paravector $x$. We denote by $\mathbb{S}^{n-1}$ the sphere of unit 1 -vectors in $\mathbb{R}^{n+1}$, i.e.

$$
\mathbb{S}^{n-1}=\left\{x=e_{1} x_{1}+\ldots+e_{n} x_{n}: x_{1}^{2}+\ldots+x_{n}^{2}=1\right\} .
$$

Any element $I \in \mathbb{S}$ is such that $I^{2}=-1$. We will denote by $\partial_{x}$ the Dirac operator $\partial_{x}=$ $\partial_{x_{0}}+e_{1} \partial_{x_{1}}+\ldots+e_{n} \partial_{x_{n}}$ and we say that a smooth function is left monogenic on the open set $U$ of $\mathbb{R}^{n+1}$ if it satisfies $\partial_{x} f(x)=0$ on $U$. In the sequel, we will denote by $\mathcal{M}(U)$ the right $\mathbb{R}_{n}$-module of (left) monogenic functions on the open set $U$ and by $\mathcal{A M}(U)$ the $\mathbb{R}_{n}$-submodule of axially monogenic functions, i.e. monogenic functions of the form $A\left(x_{0}, \rho\right)+\underline{\omega} B\left(x_{0}, \rho\right)$ with $A, B$ satisfying a Vekua-type system and $\underline{\omega} \in \mathbb{S}^{n+1}$.

Let us now recall the classical Fueter theorem for monogenic functions. Let $f$ be a holomorphic function in an open set $\mathcal{U}$ of the upper half complex plane and let

$$
f(u+\iota v)=\alpha(u, v)+\iota \beta(u, v), \quad u \in \mathbb{R}, \quad v \in \mathbb{R}^{+}
$$

where $\alpha$ and $\beta$ are differentiable functions with values in $\mathbb{R}$. The Fueter theorem in this setting states that, taken a function $f$ as above and considering the axially symmetric open set (called the open set induced by $\mathcal{U}$ )

$$
U=\left\{x=x_{0}+\underline{x} \in \mathbb{R}^{n+1}\left|x_{0}+\iota\right| \underline{x} \mid \in \mathcal{U}\right\},
$$

by replacing $u$ by $x_{0}, v$ by $|\underline{x}|$ and $\iota$ by $\underline{x} /|\underline{x}|$, we get that the function

$$
\Delta_{x}^{\frac{n-1}{2}}\left(\alpha\left(x_{0},|\underline{x}|\right)+\frac{\underline{x}}{|\underline{x}|} \beta\left(x_{0},|\underline{x}|\right)\right)
$$

where $\Delta_{x}$ is the Laplace operator in dimension $n+1$, is a monogenic function on $U$. For sake of simplicity, we will use the notation of cylindrical coordinates, i.e. we will write $x=x_{0}+I \rho$ where $\rho=|\underline{x}|, I=\frac{\underline{x}}{|\underline{x}|}$. In the sequel, taken two open sets $\mathcal{U}$ and $U$ as above, it will be useful to consider the following set of functions:

$$
\begin{gathered}
\mathcal{N}(U)=\left\{f: U \subseteq \mathbb{R}^{n+1} \rightarrow \mathbb{R}_{n}, f(x)=f\left(x_{0}+I|\underline{x}|\right)=\alpha\left(x_{0},|\underline{x}|\right)+I \beta\left(x_{0},|\underline{x}|\right) \mid\right. \\
\alpha(u, v)+\iota \beta(u, v) \text { is a } \mathbb{C} \text {-valued holomorphic function in } u+\iota v \in \mathcal{U}\} .
\end{gathered}
$$

Note that when considering functions in $\mathcal{N}(U)$, sometimes called holomorphic functions of a paravector variable, $\alpha$ and $\beta$ are $\mathbb{R}$-valued functions. However one can consider a more general 
class of functions: let $f: U \subset \mathbb{R}^{n+1} \rightarrow \mathbb{R}_{n}$ be of the form $f(x)=f\left(x_{0}+I|\underline{x}|\right)=\alpha\left(x_{0},|\underline{x}|\right)+$ $I \beta\left(x_{0},|\underline{x}|\right)$, where $I \in \mathbb{S}, \alpha$ and $\beta$ are $\mathbb{R}_{n}$-valued functions satisfying the Cauchy-Riemann system and suitable additional conditions. Note that such a function $f$ is slice monogenic in the sense of [7], (for more detail on slice monogenic functions and some of their applications see [2], [3], [5], [8], [9], [10], [11]). In the recent paper [6] we have proved an integral representation formula for $f\left(x_{0}, \rho\right)$ in terms of the function $\breve{f}\left(x_{0}, \rho\right)$, where $\breve{f}\left(x_{0}, \rho\right)$ and $f\left(x_{0}, \rho\right)$ are related by

$$
\breve{f}\left(x_{0}, \rho\right)=\Delta_{x}^{(n-1) / 2} f\left(x_{0}, \rho\right) .
$$

We have proved that the function $\breve{f}\left(x_{0}, \rho\right)$ is axially monogenic (see [12]) and that $\Delta^{(n-1) / 2}$ is surjective onto $\mathcal{A M}(U)$. Moreover, given $\breve{f} \in \mathcal{A M}(U)$ we construct a slice monogenic function $f$ satisfying (1), thus inverting the Fueter mapping theorem.

Remark 1.1. On an axially symmetric open set $U$, see [13] p. 310, every left monogenic function $\breve{f}$ can be written in the form $\breve{f}(x)=\sum_{k=0}^{\infty} \breve{f}_{k}(x)$ where $\breve{f}_{k}(x)$ are axially monogenic functions of degree $k$, i.e. $\breve{f}_{k}(x)$ are functions of the form

$$
\breve{f}_{k}(x)=A_{k}\left(x_{0}, \rho, \underline{\omega}\right)+\underline{\omega} B_{k}\left(x_{0}, \rho, \underline{\omega}\right)
$$

where $A_{k}\left(x_{0}, \rho, \underline{\omega}\right)$ and $B_{k}\left(x_{0}, \rho, \underline{\omega}\right)$ satisfy the Vekua-type system:

$$
\left\{\begin{aligned}
\partial_{x_{0}} A_{k}-\partial_{\rho} B_{k} & =\frac{k+n-1}{\rho} B_{k} \\
\partial_{x_{0}} B_{k}+\partial_{\rho} A_{k} & =\frac{k}{\rho} A_{k}
\end{aligned}\right.
$$

Even though the following definition is very well known, we recall it for sake of completeness.

Definition 1.2. A left monogenic polynomial $\mathcal{P}_{k}$ in $\mathbb{R}^{n+1}$ (resp. $\mathbb{R}^{n}$ ) is called inner spherical monogenic polynomial of degree $k$ if it is homogeneous of degree $k$, that is $\mathcal{P}_{k}(x /|x|)|x|^{k}$, (resp. $\left.\mathcal{P}_{k}(\underline{x} /|\underline{x}|)|\underline{x}|^{k}\right)$ and satisfies $\partial_{x} \mathcal{P}_{k}(x)=0\left(\operatorname{resp} . \partial_{\underline{x}} \mathcal{P}_{k}(\underline{x})=0\right)$.

Remark 1.3. For any $\left(x_{0}, \rho\right)$ fixed the functions $A_{k}$ and $B_{k}$ are inner spherical monogenics of degree $k$, see [1]. Thus we can write

$$
A_{k}\left(x_{0}, \rho, \underline{\omega}\right)=A\left(x_{0}, \rho\right) \mathcal{P}_{k}(\underline{\omega}), \quad B_{k}\left(x_{0}, \rho, \underline{\omega}\right)=B\left(x_{0}, \rho\right) \mathcal{P}_{k}(\underline{\omega})
$$

where $A\left(x_{0}, \rho\right), B\left(x_{0}, \rho\right)$ are real valued. Note that this assumption is not a restriction, in fact, in view of the finite dimensionality of the space of inner spherical monogenics we can always decompose any axially monogenic function of degree $k$ of the form $A_{k}\left(x_{0}, \rho, \underline{\omega}\right)+\underline{\omega} B_{k}\left(x_{0}, \rho, \underline{\omega}\right)$ into a finite sum of functions of the form (3), i. e.

$$
A_{k}\left(x_{0}, \rho, \underline{\omega}\right)+\underline{\omega} B_{k}\left(x_{0}, \rho, \underline{\omega}\right)=\left(A\left(x_{0}, \rho\right)+\underline{\omega} B\left(x_{0}, \rho\right)\right) \mathcal{P}_{k}(\underline{\omega}) .
$$

The preceding discussion leads to the following result (see [13]):

Theorem 1.4. Let $U \subseteq \mathbb{R}^{n+1}$ be an open set invariant with respect to $S O(n)$. Then every monogenic function $\breve{f}: \bar{U} \rightarrow \mathbb{R}_{n}$ can be written in the form $\breve{f}(x)=\sum_{k=0}^{\infty} \breve{f}_{k}(x)$ with

$$
\breve{f}_{k}(x)=\sum_{j=0}^{m_{k}}\left[A_{k, j}\left(x_{0}, \rho\right)+\underline{\omega} B_{k, j}\left(x_{0}, \rho\right)\right] \mathcal{P}_{k, j}(\underline{\omega})
$$

where $\mathcal{P}_{k, j}$ form a basis for the space of spherical monogenics of degree $k$ of dimension $m_{k}$ and $A_{k, j}, B_{k, j}$ are suitable real valued functions. 
The main aim of this paper is to find the inversion of the Fueter mapping theorem in the case of monogenic functions of type $\left(A_{k, j}\left(x_{0}, \rho\right)+\underline{\omega} B_{k, j}\left(x_{0}, \rho\right)\right) \mathcal{P}_{k, j}(\underline{\omega})$ by providing their so-called Fueter primitive.

Problem 1.5. Suppose that $U \subseteq \mathbb{R}^{n+1}$ be an axially symmetric domain, where $n$ in an odd number. Given the axially monogenic function of degree $k$

$$
\breve{f}(x) \mathcal{P}_{k}(\underline{x})=\left(A\left(x_{0}, \rho\right)+\underline{\omega} B\left(x_{0}, \rho\right)\right) \mathcal{P}_{k}(\underline{x})
$$

where $\mathcal{P}_{k}(\underline{x})$ is a spherical monogenic function determine a function $f\left(x_{0}, \rho\right)=\alpha\left(x_{0}, \rho\right)+$ $\underline{\omega} \beta\left(x_{0}, \rho\right) \in \mathcal{N}(U)$ such that

$$
\Delta_{x}^{k+\frac{n-1}{2}}\left(f(x) \mathcal{P}_{k}(\underline{x})\right)=\breve{f}(x) \mathcal{P}_{k}(\underline{x}) \quad \text { on } \quad U,
$$

where $\Delta_{x}$ is the Laplace operator in dimension $n+1$.

The solution of Problem 1.5 is given by the following integral representation formula for $f(x) \mathcal{P}_{k}(\underline{x})$ :

$$
\begin{gathered}
f(x) \mathcal{P}_{k}(\underline{x})=\int_{\Gamma} \mathcal{W}_{k, n}^{-}\left(\frac{x-y_{0}}{\rho}\right) \mathcal{P}_{k}\left(\frac{x-y_{0}}{\rho}\right) \rho^{2 k+n-2}\left[d y_{0} A\left(y_{0}, \rho\right)-d \rho B\left(y_{0}, \rho\right)\right] \\
-\int_{\Gamma} \mathcal{W}_{k, n}^{+}\left(\frac{x-y_{0}}{\rho}\right) \mathcal{P}_{k}\left(\frac{x-y_{0}}{\rho}\right) \rho^{2 k+n-2}\left[d y_{0} B\left(y_{0}, \rho\right)+d \rho A\left(y_{0}, \rho\right)\right]
\end{gathered}
$$

where $f(x) \mathcal{P}_{k}(\underline{x})$ is solution to the equation (5) and $\Gamma$ is a suitable regular curve. Here $\mathcal{W}_{k, n}^{+}$ and $\mathcal{W}_{k, n}^{-}$are explicit kernels that are determined in Section 4.

From (4) and the integral representation formula (6) we show that we can find a Fueter primitive for any monogenic function on an axially symmetric open set, see Corollary 5.4. Acknowledgments.

This research was supported by Research Foundation on Flanders (F.W.O. project 31506208).

\section{A restriction result for the Fueter primitive}

Definition 2.1 (Fueter's Primitive). Let $n$ be an odd number and let $U \subseteq \mathbb{R}^{n+1}$ be an axially symmetric domain. Let $\breve{f}(x) \mathcal{P}_{k}(\underline{x})=\left(A\left(x_{0}, \rho\right)+\underline{\omega} B\left(x_{0}, \rho\right)\right) \mathcal{P}_{k}(\underline{x})$ be an axially monogenic function of degree $k \in \mathbb{N}_{0}$. We say that a function $f(x) \mathcal{P}_{k}(\underline{x}), f \in \mathcal{N}(U)$ is a Fueter primitive of $\breve{f}(x) \mathcal{P}_{k}(\underline{x})$ if

$$
\Delta_{x}^{k+\frac{n-1}{2}}\left(f(x) \mathcal{P}_{k}(\underline{x})\right)=\breve{f}(x) \mathcal{P}_{k}(\underline{x}) \quad \text { on } \quad U,
$$

where $\Delta_{x}$ is the Laplace operator in dimension $n+1$.

Given any paravector $x=x_{0}+\underline{\omega} \rho$, where $\rho \neq 0$, it is obvious that $x$ belongs to the complex plane $\mathbb{C}_{\underline{\omega}}$ and that any paravector on the real axis belong to $\mathbb{C}_{\underline{\omega}}$ for all $\underline{\omega} \in \mathbb{S}^{n+1}$.

Definition 2.2. Let $U \subseteq \mathbb{R}^{n+1}$ be a domain. We say that $U$ is a slice domain (s-domain for short) if $U \cap \mathbb{R}$ is non empty and if $U \cap \mathbb{C}_{\underline{\omega}}$ is a domain in $\mathbb{C}_{\underline{\omega}}$ for all $\underline{\omega} \in \mathbb{S}^{n+1}$.

Remark 2.3. Let $U \subseteq \mathbb{R}^{n+1}$ be an axially symmetric s-domain. Suppose that $W \in \mathcal{N}(U)$. So function $W$ admits the power series expansion and we have

$$
W(x)=\sum_{\ell \geq 0} \frac{1}{\ell !} \underline{x}^{\ell} V^{(\ell)}\left(x_{0}\right),
$$


where $V\left(x_{0}\right):=\left.W(x)\right|_{x=0}$. For functions $W \in \mathcal{N}(U)$ the terms $V^{(\ell)}\left(x_{0}\right)$ are real numbers. The convergence is in a suitable ball $B\left(x_{0}, r\right)$ centered at $x_{0} \in U \cap \mathbb{R}$ and radius $r>0$. Finally observe that the product $W(x) \mathcal{P}_{k}(\underline{x})$ is well defined and we can write

$$
W(x) \mathcal{P}_{k}(\underline{x})=\sum_{\ell \geq 0} \frac{1}{\ell !} \underline{x}^{\ell} V^{(\ell)}\left(x_{0}\right) \mathcal{P}_{k}(\underline{x}) .
$$

We have the following results which will be crucial in the sequel. We reason in the ball $B\left(x_{0}, r\right)$ of convergence and for the Identity Principle for slice monogenic functions, see [7], the result can be extended to the whole axially symmetric s-domain $U$.

Proposition 2.4. Let $U$ be an axially symmetric s-domain in $\mathbb{R}^{n+1}, n$ be an odd number, and suppose that $W \in \mathcal{N}(U)$. Let $\mathcal{P}_{k}(\underline{x})$ be an inner left spherical monogenic polynomial of degree $k \in \mathbb{N}_{0}$. Let $x_{0} \in U \cap \mathbb{R}$ and suppose that (7) is the power series expansion of $W$ in $B\left(x_{0}, r\right)$. Then there exists a positive constant $\mathcal{H}_{k, n}$, independent of $x_{0}$, such that, for $\underline{x} \rightarrow \underline{0}$

$$
\Delta_{x}^{k+\frac{(n-1)}{2}}\left(W(x) \mathcal{P}_{k}(\underline{x})\right)=\mathcal{H}_{n, k} V^{(2 k+n-1)}\left(x_{0}\right) \mathcal{P}_{k}(\underline{x})+\mathcal{R}\left(x_{0}, \underline{x}\right) \mathcal{P}_{k}(\underline{x}),
$$

where

$$
\mathcal{H}_{k, n}:=\sum_{j=0}^{k+\frac{n-1}{2}}\left(\begin{array}{c}
k+\frac{n-1}{2} \\
j
\end{array}\right)(-1)^{j} 2^{2 j} j ! \frac{1}{(2 j) !} \frac{\Gamma\left(\frac{2 k+n}{2}+j\right)}{\Gamma\left(\frac{2 k+n}{2}\right)},
$$

and

$$
\lim _{\underline{x \rightarrow 0}} \mathcal{R}\left(x_{0}, \underline{x}\right)=0 .
$$

Proof. We set for simplicity $m=k+(n-1) / 2$ and we calculate $\Delta_{x}^{m}\left(W(x) \mathcal{P}_{k}(\underline{x})\right)$, keeping in mind that we have to take the limit $\underline{x} \rightarrow \underline{0}$. Since $\Delta_{x}=\partial_{x_{0}}^{2}+\Delta_{\underline{x}}$, we can write

$$
\begin{gathered}
\Delta_{x}^{m}\left(W(x) \mathcal{P}_{k}(\underline{x})\right)=\sum_{j=0}^{m} \sum_{\ell \geq 0}\left(\begin{array}{c}
m \\
j
\end{array}\right) \frac{1}{\ell !} \partial_{x_{0}}^{2(m-j)} \Delta_{\underline{x}}^{j}\left(\underline{x}^{\ell} \mathcal{P}_{k}(\underline{x}) V^{(\ell)}\left(x_{0}\right)\right) \\
=\sum_{j=0}^{m} \sum_{\ell \geq 0}\left(\begin{array}{c}
m \\
j
\end{array}\right) \frac{1}{\ell !} \Delta_{\underline{x}}^{j}\left(\underline{x}^{\ell} \mathcal{P}_{k}(\underline{x})\right) \partial_{x_{0}}^{2(m-j)} V^{(\ell)}\left(x_{0}\right)
\end{gathered}
$$

and we observe that

$$
\Delta_{\underline{x}}^{j}\left(\underline{x}^{\ell} \mathcal{P}_{k}(\underline{x})\right)=\left\{\begin{array}{l}
0 \quad \text { if } 2 j>\ell, \\
C_{\ell} \mathcal{P}_{k}(\underline{x}) \text { if } 2 j=\ell, \\
\mathcal{E}(\underline{x}) \mathcal{P}_{k}(\underline{x}) \text { if } 2 j<\ell,
\end{array}\right.
$$

where $C_{\ell}$ are constants depending on $\ell$ and $\mathcal{E}$ is a continuous function such that $\mathcal{E}(\underline{x}) \rightarrow 0$ for $\underline{x} \rightarrow \underline{0}$. Moreover, it is easy to see that all the terms corresponding to $2 j=\ell$ contain

$$
\partial_{x_{0}}^{2(m-j)} V^{(2 j)}\left(x_{0}\right)=V^{(2 m)}\left(x_{0}\right) .
$$

So we have

$$
\Delta_{x}^{m}\left(W(x) \mathcal{P}_{k}(\underline{x})\right)=\sum_{j=0}^{m} \sum_{\ell \geq 0}\left(\begin{array}{c}
m \\
j
\end{array}\right) \frac{1}{\ell !} \Delta_{\underline{x}}^{j}\left(\underline{x}^{\ell} \mathcal{P}_{k}(\underline{x})\right) \partial_{x_{0}}^{2(m-j)} V^{(\ell)}\left(x_{0}\right)
$$




$$
=\sum_{j=0}^{m} \frac{1}{(2 j) !}\left(\begin{array}{c}
m \\
j
\end{array}\right) \Delta_{\underline{x}}^{j}\left(\underline{x}^{2 j} \mathcal{P}_{k}(\underline{x})\right) V^{(2 m)}\left(x_{0}\right)+\mathcal{R}\left(x_{0}, \underline{x}\right) \mathcal{P}_{k}(\underline{x}) .
$$

Let us set:

$$
\mathcal{H}_{n, k}:=\sum_{j=0}^{m} \frac{1}{(2 j) !}\left(\begin{array}{c}
m \\
j
\end{array}\right) \Delta_{\underline{x}}^{j}\left(\underline{x}^{2 j} \mathcal{P}_{k}(\underline{x})\right), \quad \text { where } \quad m=k+\frac{(n-1)}{2} .
$$

Now recall that $\Delta_{\underline{x}}=-\partial_{\underline{x}}^{2}$ where $\partial_{\underline{x}}$ is the Dirac operator in dimension $n$ and the well known relations

$$
\begin{gathered}
\partial_{\underline{x}}\left(\underline{x}^{2 s} \mathcal{P}_{k}(\underline{x})\right)=-2 s \underline{x}^{2 s-1} \mathcal{P}_{k}(\underline{x}), \\
\partial_{\underline{x}}\left(\underline{x}^{2 s+1} \mathcal{P}_{k}(\underline{x})\right)=-(2 s+2 k+n) \underline{x}^{2 s} \mathcal{P}_{k}(\underline{x}) .
\end{gathered}
$$

Observe that $\Delta_{\underline{x}}^{0}\left(\underline{x}^{0} \mathcal{P}_{k}(\underline{x})\right)=1$ and consider the terms:

$$
\begin{gathered}
\Delta^{j}\left(\underline{x}^{2 j} \mathcal{P}_{k}(\underline{x})\right)=-\Delta^{j-1} \partial_{\underline{x}}^{2}\left(\underline{x}^{2 j} \mathcal{P}_{k}(\underline{x})\right)= \\
=\Delta^{j-1} \partial_{\underline{x}}\left(2 j \underline{x}^{2 j-1} \mathcal{P}_{k}(\underline{x})\right)=-2 j(2 j-2+2 k+n) \Delta^{j-1}\left(\underline{x}^{2(j-1)} \mathcal{P}_{k}(\underline{x})\right) .
\end{gathered}
$$

So we get

$$
\begin{gathered}
\Delta\left(\underline{x}^{2} \mathcal{P}_{k}(\underline{x})\right)=-2(2 k+n) \mathcal{P}_{k}(\underline{x}), \\
\Delta^{2}\left(\underline{x}^{4} \mathcal{P}_{k}(\underline{x})\right)=2 \cdot 4(2+2 k+n)(2 k+n) \mathcal{P}_{k}(\underline{x}),
\end{gathered}
$$

and by induction we have

$$
\Delta^{j}\left(\underline{x}^{2 j} \mathcal{P}_{k}(\underline{x})\right)=(-1)^{j} 2^{2 j} j ! \frac{\Gamma\left(\frac{2 k+n}{2}+j\right)}{\Gamma\left(\frac{2 k+n}{2}\right)} \mathcal{P}_{k}(\underline{x})
$$

we finally obtain that

$$
\mathcal{H}_{k, n}:=\sum_{j=0}^{m} \frac{1}{(2 j) !}\left(\begin{array}{c}
m \\
j
\end{array}\right)(-1)^{j} 2^{2 j} j ! \frac{\Gamma\left(\frac{2 k+n}{2}+j\right)}{\Gamma\left(\frac{2 k+n}{2}\right)} .
$$

This concludes the proof recalling that $m=k+\frac{(n-1)}{2}$.

\section{The kernels $\mathcal{F}_{k, n}^{+}(x)$ and $\mathcal{F}_{k, n}^{-}(x)$ and their factorization}

Using the monogenic Cauchy kernel and the inner left spherical monogenic polynomials $\mathcal{P}_{k}(\underline{x})$ we define two important kernels that we will use in the sequel. We start by recalling:

Definition 3.1 (The monogenic Cauchy kernel). We denote by $\mathcal{G}$ the monogenic Cauchy kernel on $\mathbb{R}^{n+1}$

$$
\mathcal{G}(x)=\frac{1}{A_{n+1}} \frac{\bar{x}}{|x|^{n+1}}, \quad x \in \mathbb{R}^{n+1} \backslash\{0\},
$$

where $A_{n+1}$ is the area of the unit sphere:

$$
A_{n+1}=\frac{2 \pi^{(n+1) / 2}}{\Gamma\left(\frac{n+1}{2}\right)} .
$$


Definition 3.2 (The kernels $\mathcal{F}_{k, n}^{+}(x)$ and $\mathcal{F}_{k, n}^{-}(x)$ ). Let $\mathcal{G}(x-\underline{y})$ be the monogenic Cauchy kernel defined in (9) with $x=x_{0}+\underline{x} \in \mathbb{R}^{n+1}$ and for $y=r \underline{\omega} \in \mathbb{R}^{n}$ we assume $r=1$ and $\underline{\omega} \in \mathbb{S}^{n-1}$. Let $\mathcal{P}_{k}(\underline{x})$ be an inner left spherical monogenic polynomial of degree $k \in \mathbb{N}_{0}$. We define the kernels

$$
\mathcal{F}_{k, n}^{+}(x)=\int_{\mathbb{S}^{n-1}} \mathcal{G}(x-\underline{\omega}) \mathcal{P}_{k}(\underline{\omega}) d S(\underline{\omega}), \quad \mathcal{F}_{k, n}^{-}(x)=\int_{\mathbb{S}^{n-1}} \mathcal{G}(x-\underline{\omega}) \underline{\omega} \mathcal{P}_{k}(\underline{\omega}) d S(\underline{\omega}),
$$

where $d S(\underline{\omega})$ is the scalar element of surface area of $\mathbb{S}^{n-1}$.

Before we are able to prove the main result of this section that is the factorization property of the kernels $\mathcal{F}_{k, n}^{+}(x)$ and $\mathcal{F}_{k, n}^{-}(x)$ we recall some results that we will use in the sequel.

Theorem 3.3 (Funk-Hecke (see [16])). Denote by $\mathbb{S}^{n-1}$ the unit sphere in $\mathbb{R}^{n}$ and by $A_{n-1}$ its area. Let $\xi$ and $\eta$ be two unit vectors in $\mathbb{R}^{n}$. Let $\psi$ be a real-valued function whose domain contains $[-1,1]$ and let $\mathcal{P}_{k}(\xi)$ be spherical harmonics, of degree $k$. Then we have

$$
\int_{\mathbb{S}^{n-1}} \psi(\langle\xi, \eta\rangle) \mathcal{P}_{k}(\eta) d S(\eta)=A_{n-1} \mathcal{P}_{k}(\xi) \int_{-1}^{1} \psi(t) P_{k, n}(t)\left(1-t^{2}\right)^{(n-3) / 2} d t,
$$

where $d S(\eta)$ is the scalar element of surface area on $\mathbb{S}^{n-1}$, by $\langle\xi, \eta\rangle$ we denote the scalar product of $\xi, \eta$. The Legendre polynomials are denoted by $P_{k, n}(t)$ and

$$
A_{n-1}=\frac{2 \pi^{(n-1) / 2}}{\Gamma\left(\frac{n-1}{2}\right)} .
$$

Remark 3.4. By the Rodriguez formula the Legendre polynomials $P_{k, n}(t)$ can be expressed by

$$
P_{k, n}(t)=\left(-\frac{1}{2}\right)^{k} \frac{\Gamma((n-1) / 2)}{\Gamma(k+(n-1) / 2)}\left(1-t^{2}\right)^{(3-n) / 2} \frac{d^{n}}{d t^{n}}\left(1-t^{2}\right)^{k+(n-3) / 2} .
$$

We recall the following formula (see [16] p. 188).

Proposition 3.5. Let $f:[-1,1] \rightarrow \mathbb{R}$ be a continuous function with its $n$ derivatives, then the following formula holds:

$$
\int_{-1}^{1} f(t) P_{k, n}(t)\left(1-t^{2}\right)^{(n-3) / 2} d t=\left(\frac{1}{2}\right)^{k} \frac{\Gamma((n-1) / 2)}{\Gamma(k+(n-1) / 2)} \int_{-1}^{1}\left(1-t^{2}\right)^{k+(n-3) / 2} f^{(n)}(t) d t,
$$

where $P_{k, n}$ are the Legendre polynomials, $n$ is the dimension and $k$ is the degree of $P_{n, k}$.

Theorem 3.6 (Factorization property of the kernels $\mathcal{F}_{k, n}^{+}(x)$ and $\mathcal{F}_{k, n}^{-}(x)$ ). Let $n$ be an odd number. Let $\mathcal{P}_{k}(\underline{x})$ be an inner left spherical monogenic polynomial of degree $k \in \mathbb{N}_{0}$. Let $\mathcal{F}_{k, n}^{+}(x)$ and $\mathcal{F}_{k, n}^{-}(x)$ be the kernels defined in (10). Then there exists two functions $\mathcal{S}_{k, n}^{+}(x)$ and $\mathcal{S}_{k, n}^{-}(x)$ belonging to $\mathcal{N}(U)$, independent of $\mathcal{P}_{k}(\underline{x})$, such that

$$
\mathcal{F}_{k, n}^{+}(x)=\mathcal{S}_{k, n}^{+}(x) \mathcal{P}_{k}(\underline{x}), \quad \mathcal{F}_{k, n}^{-}(x)=\mathcal{S}_{k, n}^{-}(x) \mathcal{P}_{k}(\underline{x})
$$

and

$$
\lim _{\underline{x} \rightarrow \underline{0}} \mathcal{S}_{k, n}^{+}(x)=\mathcal{C}_{k, n} \frac{x_{0}}{\left(x_{0}^{2}+1\right)^{k+(n+1) / 2}}, \quad \lim _{\underline{x} \rightarrow \underline{0}} \mathcal{S}_{k, n}^{-}(x)=-\mathcal{C}_{k, n} \frac{1}{\left(x_{0}^{2}+1\right)^{k+(n+1) / 2}},
$$

where

$$
\mathcal{C}_{k, n}:=\frac{(-1)^{k}}{\sqrt{\pi}} \frac{\Gamma\left(k+\frac{n+1}{2}\right)}{\Gamma\left(k+\frac{n}{2}\right)}
$$


Proof. Let us consider first the function $\mathcal{F}_{k, n}^{+}(x)$, for all $n$ odd number, and recalling (9), we can write $\mathcal{F}_{k, n}^{+}(x)$ as:

$$
\mathcal{F}_{k, n}^{+}(x)=\frac{1}{A_{n+1}} \int_{\mathbb{S}^{n-1}} \frac{x_{0}-\underline{x}+\underline{\omega}}{\left(x_{0}^{2}+\langle\underline{x}-\underline{\omega}, \underline{x}-\underline{\omega}\rangle\right)^{(n+1) / 2}} \mathcal{P}_{k}(\underline{\omega}) d S(\underline{\omega}) .
$$

By setting $r=|\underline{x}|, I=\underline{x} / r$, we split it as

$$
\mathcal{F}_{k, n}^{+}(x)=\left(x_{0}-\underline{x}\right) \mathcal{J}_{k, n}\left(x_{0}, r\right)+\mathcal{L}_{k, n}\left(x_{0}, r\right)
$$

where we set

$$
\begin{aligned}
\mathcal{J}_{k, n}\left(x_{0}, r\right) & :=\frac{1}{A_{n+1}} \int_{\mathbb{S}^{n-1}} \psi_{r}(t) \mathcal{P}_{k}(\underline{\omega}) d S(\underline{\omega}), \\
\mathcal{L}_{k, n}\left(x_{0}, r\right) & :=\frac{1}{A_{n+1}} \int_{\mathbb{S}^{n-1}} \psi_{r}(t) \underline{\omega} \mathcal{P}_{k}(\underline{\omega}) d S(\underline{\omega})
\end{aligned}
$$

and

$$
\psi_{r}(t):=\frac{1}{\left(x_{0}^{2}+1+r^{2}-2 r t\right)^{(n+1) / 2}}, \quad \text { where } \quad t:=\langle I, \underline{\omega}\rangle,
$$

To compute $\mathcal{F}_{k, n}^{+}(x)$ we proceed by steps.

First we calculate $\mathcal{J}_{k, n}\left(x_{0}, r\right)$ and $\mathcal{L}_{k, n}\left(x_{0}, r\right)$ by Theorem 3.3 (Funk-Hecke).

We have

$$
\mathcal{J}_{k, n}\left(x_{0}, r\right)=\frac{A_{n-1}}{A_{n+1}} \mathcal{P}_{k}(I) \int_{-1}^{1} \psi_{r}(t) P_{k, n}(t)\left(1-t^{2}\right)^{(n-3) / 2} d t
$$

and

$$
\mathcal{L}_{k, n}\left(x_{0}, r\right)=\frac{A_{n-1}}{A_{n+1}} I \mathcal{P}_{k}(I) \int_{-1}^{1} \psi_{r}(t) P_{k+1, n}(t)\left(1-t^{2}\right)^{(n-3) / 2} d t
$$

If we set

$$
\mathcal{Q}_{k, n}\left(x_{0}, r\right):=\frac{A_{n-1}}{A_{n+1}} \int_{-1}^{1} \psi_{r}(t) P_{k, n}(t)\left(1-t^{2}\right)^{(n-3) / 2} d t,
$$

then we can write $\mathcal{J}_{k, n}\left(x_{0}, r\right)$ and $\mathcal{L}_{k, n}\left(x_{0}, r\right)$ as

$$
\mathcal{J}_{k, n}\left(x_{0}, r\right)=\mathcal{Q}_{k, n}\left(x_{0}, r\right) \mathcal{P}_{k}(I)
$$

and

$$
\mathcal{L}_{k, n}\left(x_{0}, r\right)=\mathcal{Q}_{k+1, n}\left(x_{0}, r\right) I \mathcal{P}_{k}(I),
$$

so we obtain

$$
\mathcal{F}_{k, n}^{+}(x)=\left(\frac{\left(x_{0}-\underline{x}\right)}{r^{k}} \mathcal{Q}_{k, n}\left(x_{0}, r\right)+\frac{\underline{x}}{r^{k+1}} \mathcal{Q}_{k+1, n}\left(x_{0}, r\right)\right) \mathcal{P}_{k}(\underline{x})=\mathcal{S}_{k, n}^{+}(x) \mathcal{P}_{k}(\underline{x}) .
$$

This proofs the factorization of $\mathcal{F}_{k, n}^{+}(x)$ given in (12) and that, by its definition, $\mathcal{S}_{k, n}^{+}(x) \in \mathcal{N}(U)$.

Let us now calculate the limit

$$
\lim _{r \rightarrow 0}\left(\frac{\left(x_{0}-\underline{x}\right)}{r^{k}} \mathcal{Q}_{k, n}\left(x_{0}, r\right)+\frac{\underline{x}}{r^{k+1}} \mathcal{Q}_{k+1, n}\left(x_{0}, r\right)\right)
$$

where the non trivial term is only

$$
\lim _{r \rightarrow 0} \frac{x_{0}}{r^{k}} \mathcal{Q}_{k, n}\left(x_{0}, r\right) .
$$


To this end, we must study the function $\mathcal{Q}_{k, n}\left(x_{0}, r\right)$ defined in (18) for $r \rightarrow 0$.

First of all, we expand in power series the function $\psi_{r}(t)$ defined in (15) using the binomial series

$$
\psi_{r}(t)=\frac{1}{\left(x_{0}^{2}+1+r^{2}\right)^{(n+1) / 2}} \sum_{j=0}^{\infty}\left(\begin{array}{c}
-(n+1) / 2 \\
j
\end{array}\right)\left(\frac{2 r t}{x_{0}^{2}+1+r^{2}}\right)^{j} ;
$$

using the orthogonality properties of the Legendre polynomials we get

$$
\begin{gathered}
\mathcal{Q}_{k, n}\left(x_{0}, r\right)=\frac{A_{n-1}}{A_{n+1}} \frac{1}{\left(x_{0}^{2}+1+r^{2}\right)^{(n+1) / 2}}\left(\begin{array}{c}
-(n+1) / 2 \\
k
\end{array}\right)\left(\frac{2 r}{x_{0}^{2}+1+r^{2}}\right)^{k} \\
\times \int_{-1}^{1} t^{k} P_{k, n}(t)\left(1-t^{2}\right)^{(n-3) / 2} d t \\
=\frac{A_{n-1}}{A_{n+1}} 2^{k} \frac{r^{k}}{\left(x_{0}^{2}+1+r^{2}\right)^{k+(n+1) / 2}}\left(\begin{array}{c}
-(n+1) / 2 \\
k
\end{array}\right) \int_{-1}^{1} t^{k} P_{k, n}(t)\left(1-t^{2}\right)^{(n-3) / 2} d t .
\end{gathered}
$$

To explicitly compute the last integral, we use formula (21) and the well known (see [17]) integral

$$
\int_{-1}^{1}\left(1-t^{2}\right)^{q} d t=\sqrt{\pi} \frac{\Gamma(q+1)}{\Gamma\left(q+\frac{3}{2}\right)} \quad \text { for } \quad q \in \mathbb{R}^{+},
$$

so we get

$$
\begin{aligned}
\int_{-1}^{1} t^{k} P_{k, n}(t)\left(1-t^{2}\right)^{(n-3) / 2} d t & =\frac{1}{2^{k}} k ! \sqrt{\pi} \frac{\Gamma((n-1) / 2)}{\Gamma(k+(n-1) / 2)} \frac{\Gamma(1+k+(n-3) / 2)}{\Gamma(3 / 2+k+(n-2) / 2)} \\
& =\frac{1}{2^{k}} k ! \sqrt{\pi} \frac{\Gamma((n-1) / 2)}{\Gamma(k+n / 2)} .
\end{aligned}
$$

Moreover we have

$$
\left(\begin{array}{c}
-(n+1) / 2 \\
k
\end{array}\right)=\frac{(-1)^{k}}{k !} \frac{\Gamma\left(\frac{n+1}{2}+k\right)}{\Gamma\left(\frac{n+1}{2}\right)}
$$

which follows from

$$
\begin{gathered}
\left(\begin{array}{c}
-(n+1) / 2 \\
k
\end{array}\right)=\frac{1}{k !}\left(-\frac{n+1}{2}\right)\left(-\frac{n+3}{2}\right) \ldots\left(-\frac{n+2 k-1}{2}\right) \\
=\frac{(-1)^{k}}{k !}\left(\frac{n+1}{2}\right)\left(\frac{n+1}{2}+1\right)\left(\frac{n+1}{2}+2\right) \ldots\left(\frac{n+1}{2}+k-1\right)=\frac{(-1)^{k}}{k !} \frac{\Gamma\left(\frac{n+1}{2}+k\right)}{\Gamma\left(\frac{n+1}{2}\right)} .
\end{gathered}
$$

Finally we get

$$
\begin{gathered}
\mathcal{Q}_{k, n}\left(x_{0}, r\right)=\frac{A_{n-1}}{A_{n+1}} 2^{k} \frac{r^{k}}{\left(x_{0}^{2}+1+r^{2}\right)^{k+(n+1) / 2}}\left(\begin{array}{c}
-(n+1) / 2 \\
k
\end{array}\right) \int_{-1}^{1} t^{k} P_{k, n}(t)\left(1-t^{2}\right)^{(n-3) / 2} d t \\
=\frac{A_{n-1}}{A_{n+1}} 2^{k} \frac{r^{k}}{\left(x_{0}^{2}+1+r^{2}\right)^{k+(n+1) / 2}} \times(-1)^{k} \frac{\Gamma\left(\frac{n+1}{2}+k\right)}{k ! \Gamma\left(\frac{n+1}{2}\right)} \times \frac{1}{2^{k}} k ! \sqrt{\pi} \frac{\Gamma((n-1) / 2)}{\Gamma(k+n / 2)}
\end{gathered}
$$

with some simplifications we obtain

$$
\mathcal{Q}_{k, n}\left(x_{0}, r\right)=\mathcal{C}_{k, n} \frac{r^{k}}{\left(x_{0}^{2}+1+r^{2}\right)^{k+(n+1) / 2}}
$$


where

$$
\mathcal{C}_{k, n}:=\frac{(-1)^{k}}{\sqrt{\pi}} \frac{\Gamma\left(k+\frac{n+1}{2}\right)}{\Gamma\left(k+\frac{n}{2}\right)} .
$$

Finally, we compute

$$
\lim _{r \rightarrow 0} \frac{x_{0}}{r^{k}} \mathcal{Q}_{k, n}\left(x_{0}, r\right)=\mathcal{C}_{k, n} \frac{x_{0}}{\left(x_{0}^{2}+1\right)^{k+(n+1) / 2}},
$$

from which we deduce the limit in (13), that is $\lim _{\underline{x} \rightarrow \underline{0}} \mathcal{S}_{k, n}^{+}(x)$. The above computations allows us to determine also the factorization for $\mathcal{F}_{k, n}^{-}(x)$ and the limit for $\mathcal{S}_{k, n}^{-}(x)$. In fact

$$
\begin{gathered}
\mathcal{F}_{k, n}^{-}(x)=\frac{1}{A_{n+1}} \int_{\mathbb{S}^{n-1}} \frac{x_{0}-\underline{x}+\underline{\omega}}{\left(x_{0}^{2}+\langle\underline{x}-\underline{\omega}, \underline{x}-\underline{\omega}\rangle\right)^{(n+1) / 2} \underline{\omega} \mathcal{P}_{k}(\underline{\omega}) d S(\underline{\omega})} \\
=\left(x_{0}-\underline{x}\right) \mathcal{L}_{k, n}\left(x_{0}, r\right)-\mathcal{J}_{k, n}\left(x_{0}, r\right) .
\end{gathered}
$$

With analogous calculations, we deduce the factorization $\mathcal{F}_{k, n}^{-}(x)=\mathcal{S}_{k, n}^{-}(x) \mathcal{P}_{k}(\underline{x})$ with $\mathcal{S}_{k, n}^{-}(x) \in$ $\mathcal{N}(U)$ and the limit

$$
\lim _{\underline{x \rightarrow 0}} \mathcal{S}_{k, n}^{+}(x)=-\mathcal{C}_{k, n} \frac{1}{\left(x_{0}^{2}+1\right)^{k+(n+1) / 2}} .
$$

This concludes the proof.

\section{The Fueter primitives of the kernels $\mathcal{F}_{k, n}^{+}(x)$ and $\mathcal{F}_{k, n}^{-}(x)$}

The definition of Fueter's primitive and the factorization property of the kernels $\mathcal{F}_{k, n}^{+}(x)$ and $\mathcal{F}_{k, n}^{-}(x)$, see Theorem (3.6), require the determination only of the functions $\mathcal{S}_{k, n}^{+}(x)$ and $\mathcal{S}_{k, n}^{-}(x)$.

Definition 4.1. Let $n$ be an odd number. Let $\mathcal{P}_{k}(\underline{x})$ be an inner left spherical monogenic polynomial of degree $k \in \mathbb{N}_{0}$. We will denote by $\mathcal{W}_{k, n}^{+}(x) \mathcal{P}_{k}(\underline{x})$ and $\mathcal{W}_{k, n}^{-}(x) \mathcal{P}_{k}(\underline{x})$ the Fueter primitives of $\mathcal{F}_{k, n}^{+}(x)$ and $\mathcal{F}_{k, n}^{-}(x)$, that is $\mathcal{W}_{k, n}^{+}(x) \mathcal{P}_{k}(\underline{x})$ and $\mathcal{W}_{k, n}^{-}(x) \mathcal{P}_{k}(\underline{x})$ satisfying

$$
\Delta^{k+\frac{n-1}{2}}\left(\mathcal{W}_{k, n}^{+}(x) \mathcal{P}_{k}(\underline{x})\right)=\mathcal{F}_{k, n}^{+}(x), \quad \Delta^{k+\frac{n-1}{2}}\left(\mathcal{W}_{k, n}^{-}(x) \mathcal{P}_{k}(\underline{x})\right)=\mathcal{F}_{k, n}^{-}(x) .
$$

Remark 4.2. Let us observe that thanks to Theorem 3.6 we also have

$$
\Delta^{k+\frac{n-1}{2}}\left(\mathcal{W}_{k, n}^{+}(x) \mathcal{P}_{k}(\underline{x})\right)=\mathcal{S}_{k, n}^{+}(x) \mathcal{P}_{k}(\underline{x}), \quad \Delta^{k+\frac{n-1}{2}}\left(\mathcal{W}_{k, n}^{-}(x) \mathcal{P}_{k}(\underline{x})\right)=\mathcal{S}_{k, n}^{-}(x) \mathcal{P}_{k}(\underline{x}) .
$$

Theorem 4.3 (The explicit structure of the functions $\mathcal{W}_{k, n}^{+}(x)$ and $\mathcal{W}_{k, n}^{-}(x)$ ). Let $n$ be an odd number and let $k \in \mathbb{N}_{0}$. If $\mathcal{S}_{k, n}^{+}(x)$ and $\mathcal{S}_{k, n}^{-}(x)$, are the functions in (12), then we have

$$
\begin{gathered}
\mathcal{W}_{k, n}^{+}\left(x_{0}\right)=\frac{\mathcal{C}_{k, n}}{\mathcal{H}_{k, n}} D^{-(2 k+n-1)} \frac{x_{0}}{\left(x_{0}^{2}+1\right)^{k+(n+1) / 2}}, \\
\mathcal{W}_{k, n}^{-}\left(x_{0}\right)=-\frac{\mathcal{C}_{k, n}}{\mathcal{H}_{k, n}} D^{-(2 k+n-1)} \frac{1}{\left(x_{0}^{2}+1\right)^{k+(n+1) / 2}},
\end{gathered}
$$

where the symbol $D^{-(n-1+2 k)}$ stands for the $(2 k+n-1)$ integrations with respect to $x_{0}$. Replacing now $x_{0}$ by $x$ in $\mathcal{W}_{k, n}^{+}\left(x_{0}\right)$ and in $\mathcal{W}_{k, n}^{-}\left(x_{0}\right)$ we get $\mathcal{W}_{k, n}^{+}(x)$ and $\mathcal{W}_{k, n}^{-}(x)$, respectively. Moreover, the functions $\mathcal{W}_{k, n}^{+}(x)$ and $\mathcal{W}_{k, n}^{-}(x)$ belong to $\mathcal{N}(U)$. 
Proof. Let us observe that the kernels $\mathcal{S}_{k, n}^{+}(x)$ and $\mathcal{S}_{k, n}^{-}(x)$ defined in (10) are axially monogenic, they satisfy the Vekua system which is elliptic so $\mathcal{S}_{k, n}^{+}(x)$ and $\mathcal{S}_{k, n}^{-}(x)$ are determined by their restrictions to $\underline{x}=0$. From Theorem 3.6 we have that

$$
\lim _{\underline{x} \rightarrow \underline{0}} \mathcal{S}_{k, n}^{+}(x)=\mathcal{C}_{k, n} \frac{x_{0}}{\left(x_{0}^{2}+1\right)^{k+(n+1) / 2}}, \quad \lim _{\underline{x} \rightarrow \underline{0}} \mathcal{S}_{k, n}^{+}(x)=-\mathcal{C}_{k, n} \frac{1}{\left(x_{0}^{2}+1\right)^{k+(n+1) / 2}},
$$

where the constants $\mathcal{C}_{k, n}$ are explicitly determine by (14). Now recall that if $W \in \mathcal{N}(U)$, defined on an axially symmetric s-domain in $\mathbb{R}^{n+1}$, from Proposition 2.4 , recalling that $\mathcal{P}_{k}(\underline{x})$ are inner left spherical monogenic polynomials of degree $k \in \mathbb{N}_{0}$, we have that, for $\underline{x} \rightarrow \underline{0}$,

$$
\Delta_{x}^{k+(n-1) / 2}\left(W(x) \mathcal{P}_{k}(\underline{x})\right)=\mathcal{H}_{n, k} V^{(2 k+n-1)}\left(x_{0}\right) \mathcal{P}_{k}(\underline{x})+\mathcal{R}\left(x_{0}, \underline{x}\right) \mathcal{P}_{k}(\underline{x}),
$$

where $\left.W(x)\right|_{\underline{x}=0}:=V\left(x_{0}\right)$ and $\mathcal{H}_{n, k}$ are explicitly determined by (8). If we set

$$
D^{(2 k+n-1)} \mathcal{W}_{k, n}^{+}\left(x_{0}\right)=\frac{\mathcal{C}_{k, n}}{\mathcal{H}_{k, n}} \frac{x_{0}}{\left(x_{0}^{2}+1\right)^{k+(n+1) / 2}}
$$

and we integrate the function $\frac{x_{0}}{\left(x_{0}^{2}+1\right)^{k+(n+1) / 2}}$ for $(2 k+n-1)$ times we get

$$
\mathcal{W}_{k, n}^{+}\left(x_{0}\right)=\frac{\mathcal{C}_{k, n}}{\mathcal{H}_{k, n}} D^{-(2 k+n-1)} \frac{x_{0}}{\left(x_{0}^{2}+1\right)^{k+(n+1) / 2}}
$$

where the symbol $D^{-(2 k+n-1)}$ stands for the $(2 k+n-1)$ integrations with respect to $x_{0}$. By replacing now $x_{0}$ by $x$ in $\mathcal{S}_{k, n}^{+}\left(x_{0}\right)$ we get $\mathcal{S}_{k, n}^{+}(x)$. We observe that it is the required function since

$$
\begin{gathered}
\lim _{\underline{x} \rightarrow \underline{0} \Delta_{x}^{k+(n-1) / 2}}\left(\mathcal{W}_{k, n}^{+}(x) \mathcal{P}_{k}(\underline{x})\right)=\frac{\mathcal{C}_{k, n}}{\mathcal{H}_{k, n}} \mathcal{H}_{k, n} V^{(2 k+n-1)}\left(x_{0}\right) \lim _{\underline{x \rightarrow} \underline{0}} \mathcal{P}_{k}(\underline{x}) \\
=\mathcal{C}_{k, n} \frac{x_{0}}{\left(x_{0}^{2}+1\right)^{k+(n+1) / 2}} \lim _{\underline{x} \rightarrow \underline{0}} \mathcal{P}_{k}(\underline{x}) .
\end{gathered}
$$

Analogously we set

$$
D^{(2 k+n-1)} \mathcal{S}_{k, n}^{-}\left(x_{0}\right):=-\frac{\mathcal{C}_{n}}{\mathcal{K}_{n}} \frac{1}{\left(x_{0}^{2}+1\right)^{k+(n+1) / 2}}
$$

and we integrate the function $\frac{1}{\left(x_{0}^{2}+1\right)^{k+(n+1) / 2}}$ for $(2 k+n-1)$ times we get

$$
\mathcal{W}_{k, n}^{-}\left(x_{0}\right):=-\frac{\mathcal{C}_{k, n}}{\mathcal{H}_{k, n}} D^{-(2 k+n-1)} \frac{1}{\left(x_{0}^{2}+1\right)^{k+(n+1) / 2}}
$$

Replacing now $x_{0}$ by $x$ in $\mathcal{W}_{k, n}^{-}\left(x_{0}\right)$ we get $\mathcal{W}_{k, n}^{-}(x)$ which is the required function. Finally we observe that the functions

$$
x_{0} \mapsto \frac{x_{0}}{\left(x_{0}^{2}+1\right)^{k+(n+1) / 2}}, \quad x_{0} \mapsto \frac{1}{\left(x_{0}^{2}+1\right)^{k+(n+1) / 2}},
$$

can be integrated by parts in closed form an arbitrary number of times. Such primitives contain rational functions of $x_{0}$ and $\arctan x_{0}$ of the real variable $x_{0}$.

When we replace in such functions the real variable $x_{0}$ by the paravector variable $x$ we clearly obtain that the functions $\mathcal{W}_{k, n}^{-}$and $\mathcal{W}_{k, n}^{+}$belong to $\mathcal{N}(U)$. 


\section{The inverse Fueter mapping theorem in integral form}

We now recall the Cauchy's integral formula for monogenic functions that with the results of the previous section is the main tool to prove our main result.

Theorem 5.1 (Cauchy's integral representation theorem for monogenic functions). Let $\breve{f}$ be a left monogenic function in $U \subseteq \mathbb{R}^{n+1}$. Then, for every $M \subset U$ and for $x \in M$, we have

$$
\breve{f}(x)=\int_{\partial M} \mathcal{G}(y-x) d \sigma(y) \breve{f}(y)
$$

where $\partial M$ is supposed to be an n-dimensional compact smooth manifold in $U$, the differential form $d \sigma(y)$ is given by $d \sigma(y)=\eta(y) d S(y)$ where $\eta(y)$ is the outer unit normal to $\partial M$ at point $y$ and $d S(y)$ is the scalar element of surface area on $\partial M$.

We are now in position to state and prove the main result of this paper.

Theorem 5.2 (The inverse Fueter mapping theorem). Let $n$ be an odd number and let $\mathcal{P}_{k}(\underline{x})$ be an inner left spherical monogenic polynomial of degree $k \in \mathbb{N}_{0}$. Let

$$
\breve{f}(x) \mathcal{P}_{k}(\underline{x})=\left(A\left(x_{0}, \rho\right)+\underline{\omega} B\left(x_{0}, \rho\right)\right) \mathcal{P}_{k}(\underline{x})
$$

be an axially monogenic function of degree $k$ defined on an axially symmetric open set $U \subseteq \mathbb{R}^{n+1}$. Let $\Gamma$ be the boundary of an open bounded subset $\mathcal{V}$ of the half plane $\mathbb{R}+\underline{\omega} \mathbb{R}^{+}$and let $V \subset U$ be the open set in $\mathbb{R}^{n+1}$ induced by $\mathcal{V}$. Moreover suppose that $\Gamma$ is a regular curve whose parametric equations $y_{0}=y_{0}(s), \rho=\rho(s)$ are expressed in terms of the arc-length $s \in[0, L], L>0$ and consider the manifold

$$
\Sigma:=\left\{y_{0}+\underline{\omega} \rho \mid\left(y_{0}, \rho\right) \in \Gamma, \underline{\omega} \in \mathbb{S}^{n-1}\right\} .
$$

Then the function

$$
\begin{gathered}
f(x) \mathcal{P}_{k}(\underline{x})=\int_{\Gamma} \mathcal{W}_{k, n}^{-}\left(\frac{x-y_{0}}{\rho}\right) \mathcal{P}_{k}\left(\frac{x-y_{0}}{\rho}\right) \rho^{2 k+n-2}\left[d y_{0} A\left(y_{0}, \rho\right)-d \rho B\left(y_{0}, \rho\right)\right] \\
-\int_{\Gamma} \mathcal{W}_{k, n}^{+}\left(\frac{x-y_{0}}{\rho}\right) \mathcal{P}_{k}\left(\frac{x-y_{0}}{\rho}\right) \rho^{2 k+n-2}\left[d y_{0} B\left(y_{0}, \rho\right)+d \rho A\left(y_{0}, \rho\right)\right]
\end{gathered}
$$

is a Fueter's primitive of $\breve{f}(x) \mathcal{P}_{k}(\underline{x})$ on $V$.

Proof. We represent axially monogenic functions $\breve{f}$ by the Cauchy formula (22) using the manifold $\Sigma$ as follows. We specify the notations: $d s$ is the infinitesimal arc-length, $d S(\underline{\omega})$ is the infinitesimal element of surface area on $\mathbb{S}^{n-1} ; \mathbf{t}=\frac{d}{d s}\left(y_{0}+\underline{\omega} \rho\right)$ is the unit tangent vector in a point of $\Gamma$, while the normal unit vector is given by

$$
\mathbf{n}=-\underline{\omega} \mathbf{t}=\frac{d}{d s}\left[\rho(s)-\underline{\omega} y_{0}(s)\right] .
$$

The scalar infinitesimal element of the manifold $\Sigma$, express in terms of $d s$ and $d S$ is given by

$$
d \Sigma=\rho^{n-1} d s d S(\underline{\omega}),
$$

finally the oriented infinitesimal element of manifold $d \sigma(s, \underline{\omega})$ is given by

$$
d \sigma(s, \underline{\omega})=\mathbf{n} d \Sigma=\frac{d}{d s}\left[\rho(s)-\underline{\omega} y_{0}(s)\right] \rho^{n-1} d s d S(\underline{\omega})
$$


so finally we get

$$
d \sigma(s, \underline{\omega})=\left[d \rho(s)-\underline{\omega} d y_{0}(s)\right] \rho^{n-1} d S(\underline{\omega}) .
$$

Thanks to the above considerations we have:

$$
\breve{f}\left(x_{0}+I r\right) \mathcal{P}_{k}(I)=\int_{\Gamma} \int_{S^{n-1}} \mathcal{G}\left(y_{0}+\underline{\omega} \rho-x_{0}-r I\right) d \sigma(s, \underline{\omega}) \breve{f}\left(y_{0}+\underline{\omega} \rho\right) \mathcal{P}_{k}(\underline{\omega}) .
$$

So we can split the integral in the following way

$$
\begin{gathered}
\breve{f}\left(x_{0}+I r\right) \mathcal{P}_{k}(I)=-\int_{\Gamma}\left[\int_{\mathbb{S}^{n-1}} \mathcal{G}\left(y_{0}+\underline{\omega} \rho-x_{0}-r I\right) \underline{\omega} \mathcal{P}_{k}(\underline{\omega}) d S(\underline{\omega})\right] \rho^{n-1}\left[d y_{0} A\left(y_{0}, \rho\right)-d \rho B\left(y_{0}, \rho\right)\right] \\
+\int_{\Gamma}\left[\int_{S^{n-1}} \mathcal{G}\left(y_{0}+\underline{\omega} \rho-x_{0}-r I\right) \mathcal{P}_{k}(\underline{\omega}) d S(\underline{\omega})\right] \rho^{n-1}\left[d y_{0} B\left(y_{0}, \rho\right)+d \rho A\left(y_{0}, \rho\right)\right]
\end{gathered}
$$

keeping in mind the property $\mathcal{G}(t x)=t^{-n} \mathcal{G}(t x)$ for $t>0$, with a change of variables, we have

$$
\begin{gathered}
\breve{f}\left(x_{0}+\operatorname{Ir}\right) \mathcal{P}_{k}(I)=\int_{\Gamma}\left[\int_{\mathbb{S}^{n-1}} \rho^{-n} \mathcal{G}\left(\frac{x_{0}-y_{0}}{\rho}+\frac{r}{\rho} I-\underline{\omega}\right) \underline{\omega} \mathcal{P}_{k}(\underline{\omega}) d S(\underline{\omega})\right] \rho^{n-1}\left[d y_{0} A\left(y_{0}, \rho\right)-d \rho B\left(y_{0}, \rho\right)\right] \\
-\int_{\Gamma}\left[\int_{\mathbb{S}^{n-1}} \rho^{-n} \mathcal{G}\left(\frac{x_{0}-y_{0}}{\rho}+\frac{r}{\rho} I-\underline{\omega}\right) \mathcal{P}_{k}(\underline{\omega}) d S(\underline{\omega})\right] \rho^{n-1}\left[d y_{0} B\left(y_{0}, \rho\right)+d \rho A\left(y_{0}, \rho\right)\right]
\end{gathered}
$$

recalling the definitions of $\mathcal{F}_{k, n}^{+}$and $\mathcal{F}_{k, n}^{-}$we get

$$
\begin{gathered}
\breve{f}\left(x_{0}+\operatorname{Ir}\right) \mathcal{P}_{k}(I)=\int_{\Gamma} \mathcal{F}_{k, n}^{-}\left(\frac{x_{0}-y_{0}}{\rho}+\frac{r}{\rho} I\right) \rho^{-1}\left[d y_{0} A\left(y_{0}, \rho\right)-d \rho B\left(y_{0}, \rho\right)\right] \\
-\int_{\Gamma} \mathcal{F}_{k, n}^{+}\left(\frac{x_{0}-y_{0}}{\rho}+\frac{r}{\rho} I\right) \rho^{-1}\left[d y_{0} B\left(y_{0}, \rho\right)+d \rho A\left(y_{0}, \rho\right)\right] .
\end{gathered}
$$

Let us observe that, since $x=x_{0}+I r$, we have

$$
\begin{gathered}
\breve{f}\left(x_{0}+\operatorname{Ir}\right) \mathcal{P}_{k}(I)=\int_{\Gamma} \mathcal{F}_{k, n}^{-}\left(\frac{x-y_{0}}{\rho}\right) \rho^{-1}\left[d y_{0} A\left(y_{0}, \rho\right)-d \rho B\left(y_{0}, \rho\right)\right] \\
-\int_{\Gamma} \mathcal{F}_{k, n}^{+}\left(\frac{x-y_{0}}{\rho}\right) \rho^{-1}\left[d y_{0} B\left(y_{0}, \rho\right)+d \rho A\left(y_{0}, \rho\right)\right] .
\end{gathered}
$$

By setting

$$
x^{\prime}:=\frac{x-y_{0}}{\rho}
$$

by Definition (4.1), we obtain

$$
\begin{gathered}
\breve{f}\left(x_{0}+I r\right) \mathcal{P}_{k}(I)=\int_{\Gamma} \Delta_{x^{\prime}}^{k+(n-1) / 2}\left(\mathcal{W}_{k, n}^{-}\left(x^{\prime}\right) \mathcal{P}_{k}\left(\underline{x}^{\prime}\right)\right) \rho^{-1}\left[d y_{0} A\left(y_{0}, \rho\right)-d \rho B\left(y_{0}, \rho\right)\right] \\
-\int_{\Gamma} \Delta_{x^{\prime}}^{k+(n-1) / 2}\left(\mathcal{W}_{k, n}^{+}\left(x^{\prime}\right) \mathcal{P}_{k}\left(\underline{x}^{\prime}\right)\right) \rho^{-1}\left[d y_{0} B\left(y_{0}, \rho\right)+d \rho A\left(y_{0}, \rho\right)\right]
\end{gathered}
$$

and since $\Delta_{x^{\prime}}^{k+(n-1) / 2}=\rho^{2 k+n-1} \Delta_{x}^{k+(n-1) / 2}$ we get:

$$
\breve{f}\left(x_{0}+\operatorname{Ir}\right) \mathcal{P}_{k}(I)=\Delta_{x}^{k+(n-1) / 2}\left[\int_{\Gamma} \mathcal{W}_{k, n}^{-}\left(\frac{x-y_{0}}{\rho}\right) \mathcal{P}_{k}\left(\frac{x-y_{0}}{\rho}\right) \rho^{2 k+n-2}\left[d y_{0} A\left(y_{0}, \rho\right)-d \rho B\left(y_{0}, \rho\right)\right]\right.
$$




$$
\left.-\int_{\Gamma} \mathcal{W}_{k, n}^{+}\left(\frac{x-y_{0}}{\rho}\right) \mathcal{P}_{k}\left(\frac{x-y_{0}}{\rho}\right) \rho^{2 k+n-2}\left[d y_{0} B\left(y_{0}, \rho\right)+d \rho A\left(y_{0}, \rho\right)\right]\right]
$$

where

$$
\begin{gathered}
f(x) \mathcal{P}_{k}(\underline{x})=\int_{\Gamma} \mathcal{W}_{k, n}^{-}\left(\frac{x-y_{0}}{\rho}\right) \mathcal{P}_{k}\left(\frac{x-y_{0}}{\rho}\right) \rho^{2 k+n-2}\left[d y_{0} A\left(y_{0}, \rho\right)-d \rho B\left(y_{0}, \rho\right)\right] \\
-\int_{\Gamma} \mathcal{W}_{k, n}^{+}\left(\frac{x-y_{0}}{\rho}\right) \mathcal{P}_{k}\left(\frac{x-y_{0}}{\rho}\right) \rho^{2 k+n-2}\left[d y_{0} B\left(y_{0}, \rho\right)+d \rho A\left(y_{0}, \rho\right)\right] .
\end{gathered}
$$

From the proof on the above theorem one can easily see that the following result holds.

Corollary 5.3. Under the hypothesis of the above theorem, the Cauchy integral formula for axially monogenic functions $\breve{f}$ of degree $k$ can be written on $V$ in the form

$$
\begin{gathered}
\breve{f}(x) \mathcal{P}_{k}(\underline{x})=\int_{\Gamma} \mathcal{F}_{k, n}^{-}\left(\frac{x-y_{0}}{\rho}\right) \rho^{-1}\left[d y_{0} A\left(y_{0}, \rho\right)-d \rho B\left(y_{0}, \rho\right)\right] \\
-\int_{\Gamma} \mathcal{F}_{k, n}^{+}\left(\frac{x-y_{0}}{\rho}\right) \rho^{-1}\left[d y_{0} B\left(y_{0}, \rho\right)+d \rho A\left(y_{0}, \rho\right)\right] .
\end{gathered}
$$

Another consequence is the following corollary which needs some more notations in order to be stated. Let us denote by $\mathcal{A M}_{k}(U)$ the set of axially symmetric functions on the axially symmetric open set $U$ and let us introduce the set

$$
\mathcal{N}_{k}(U)=\left\{\varphi_{k}=\sum_{j=0}^{m_{k}} f_{j}(x) \mathcal{P}_{k, j}(\underline{x}) \mid f_{j} \in \mathcal{N}(U), \quad \mathcal{P}_{k, j} \text { spherical monogenic of degree } k\right\},
$$

where $m_{k}=\operatorname{dim} \mathcal{A M}_{k}$.

Corollary 5.4. Let $n$ be an odd number and let $U$ be an axially symmetric open set in $\mathbb{R}^{n+1}$. There is a map of $\mathbb{R}_{n}$-modules

$$
\mathcal{A M}_{k}(U) \rightarrow \mathcal{N}_{k}(U)
$$

such that, given $\left(A_{k}+\underline{\omega} B_{k}\right) \mathcal{P}_{k} \in \mathcal{A M}_{k}(U)$, we have $\left(A_{k}+\underline{\omega} B_{k}\right) \mathcal{P}_{k}=\Delta^{k+\frac{n-1}{2}}\left(\left(\alpha_{k}+\underline{\omega} \beta_{k}\right) \mathcal{P}_{k}\right)$, with $\alpha_{k}+\underline{\omega} \beta_{k} \in \mathcal{N}(U)$, and a map

$$
\mathcal{M}(U) \rightarrow \bigoplus_{k} \Delta^{k} \mathcal{N}_{k}(U)
$$

such that, given $\breve{f}=\sum_{k} \breve{f}_{k} \in \mathcal{M}(U), f_{k} \in \mathcal{A M}_{k}(U)$, there are $\varphi_{k} \in \mathcal{N}_{k}$ such that

$$
\breve{f}=\Delta^{\frac{n-1}{2}} \sum_{k} \Delta^{k} \varphi_{k} .
$$

Proof. It is a consequence of the inverse Fueter mapping theorem and of Theorem 1.4. 


\section{References}

[1] F. Brackx, R. Delanghe, F. Sommen, Clifford Analysis, Pitman Res. Notes in Math., 76, 1982.

[2] F. Colombo, I. Sabadini, A structure formula for slice monogenic functions and some of its consequences, Hypercomplex Analysis, Trends in Mathematics, Birkhäuser, 2009, 101-114.

[3] F. Colombo, I. Sabadini, The Cauchy formula with s-monogenic kernel and a functional calculus for noncommuting operators, to appear in J. Math. Anal. Appl. DOI: 10.1016/j.jmaa.2010.08.016.

[4] F. Colombo, I. Sabadini, F. Sommen, D.C. Struppa, Analysis of Dirac Systems and Computational Algebra, Progress in Mathematical Physics, Vol. 39, Birkhäuser, Boston, 2004.

[5] F. Colombo, I. Sabadini, F. Sommen, The Fueter mapping theorem in integral form and the $\mathcal{F}$-functional calculus, to appear in Math. Methods Appl. Sci. (2010). DOI:10.1002/mma.1315

[6] F. Colombo, I. Sabadini, F. Sommen, The inverse Fueter mapping theorem, preprint 2010.

[7] F. Colombo, I. Sabadini, D.C. Struppa, Slice monogenic functions, Israel J. Math., 171 (2009), 385-403.

[8] F. Colombo, I. Sabadini, D.C. Struppa, Extension properties for slice monogenic functions, Israel J. Math., 177 (2010), 369-389.

[9] F. Colombo, I. Sabadini, D.C. Struppa, A new functional calculus for noncommuting operators, J. Funct. Anal., 254 (2008), 2255-2274.

[10] F. Colombo, I. Sabadini, D.C. Struppa, Duality theorems for slice hyperholomorphic functions, to appear in J. Reine Angew. Math., (2010).

[11] F. Colombo, I. Sabadini, D.C. Struppa, Noncommutative Functional Calculus, preprint (2010).

[12] A. K. Common, F. Sommen, Axial monogenic functions from holomorphic functions, J. Math. Anal. Appl., 179 (1993), 610-629.

[13] R. Delanghe, F. Sommen, V. Souček, Clifford Algebra and Spinor-valued Functions, Mathematics and Its Applications 53, Kluwer Academic Publishers, 1992.

[14] R. Fueter, Die Funktionentheorie der Differentialgleichungen $\Delta u=0$ und $\Delta \Delta u=0$ mit vier reellen Variablen, Comm. Math. Helv., 7 (1934), 307-330.

[15] J. E. Gilbert, M. A. M. Murray, Clifford algebras and Dirac operators in harmonic analysis, Cambridge studies in advanced mathematics n. 26 (1991).

[16] H. Hochstadt, The functions of Mathematical Physics, Pure Appl. Math., vol 23, WileyInterscience, New York, 1971.

[17] I. S. Gradshteyn, I. M. Ryzhik, Table of Integrals, Series, and Products, Accademic Press LTD, Mathematics / Engineering, Sixth Edition (2000). 
[18] K. Gürlebeck, K. Habetha, W. Sprößig, Holomorphic Functions in the Plane and ndimensional space, Birkhäuser, Basel, 2008.

[19] K. I. Kou, T. Qian, F. Sommen, Generalizations of Fueter's theorem, Meth. Appl. Anal., 9 (2002), 273-290.

[20] D. Pena-Pena, Cauchy-Kowalevski extensions, Fueters theorems and boundary values of special systems in Clifford analysis, PhD Dissertation, Gent (2008).

[21] D. Pena-Pena, T. Qian, F. Sommen, An alternative proof of Fueter's theorem, Complex Var. Elliptic Equ., 51 (2006), 913-922.

[22] T. Qian, Generalization of Fueter's result to $\mathbb{R}^{n+1}$, Rend. Mat. Acc. Lincei, 8 (1997), 111117.

[23] T. Qian, Fourier analysis on a starlike Lipschitz aurfaces, J. Funct. Anal., 183 (2001), $370-412$.

[24] T. Qian, Singular integrals on star-shaped Lipschitz surfaces in the quaternionic space, Math. Ann., 310 (1998), 601-630.

[25] M. Sce, Osservazioni sulle serie di potenze nei moduli quadratici, Atti Acc. Lincei Rend. Fisica, 23 (1957), 220-225.

[26] F. Sommen, On a generalization of Fueter's theorem, Zeit. Anal. Anwen., 19 (2000), 899902. 